\title{
Positive set-operators of low complexity
}

\author{
Athanassios Tzouvaras
}

Dept. of Mathematics, Univ. of Thessaloniki, 54006 Thessaloniki, Greece. e-mail:tzouvara@auth.gr

Current address:

Dept. of Mathematics and Statistics,

University of Cyprus,

P.O. Box 20537

1678 Nicosia, Cyprus

e-mail:tzouvara@ucy.ac.cy

\begin{abstract}
The powerset operator, $\mathcal{P}$, is compared with other operators of similar type and logical complexity. Namely we examine positive operators whose defining formula has a canonical form containing at most a string of universal quantifiers. We call them $\forall$-operators. The question we address in this paper is: How is the class of $\forall$-operators generated? It is shown that every positive $\forall$-operator $\Gamma$ such that $\Gamma(\emptyset) \neq \emptyset$, is finitely generated from $\mathcal{P}$, the identity operator $I d$, constant operators and certain trivial ones by composition, $\cup$ and $\cap$. This extends results of [3] concerning bounded positive operators.
\end{abstract}

2000 Mathematics Subject Classification. Primary 03E05, secondary 03E20.

Key words. Powerset operator, positive set-operator, $\forall$-operator. 


\section{Introduction}

$\mathcal{P}$ is a very special operator in set theory. On the one hand its iterations along the ordinals create the entire universe. On the other hand $\mathcal{P}$ is relative, to the effect that many people wonder whether, for infinite $X$, the objects $\mathcal{P}(X)$ (among them the universe $V$ as well as the set $\mathbb{R}$ of real numbers) are well-determined and definite (cf. [1] for a recent discussion on the issue). In [3] we started an investigation of the main features of $\mathcal{P}$, with the aim to detect all those operators that share these features. The main properties of $\mathcal{P}$ are: (1) It is settheoretic, i.e., it sends sets to sets (as a consequence of the powerset axiom), (2) it is positive, i.e., defined by a positive formula (hence monotone) (3) it is cardinality raising, i.e., $|x|<|\mathcal{P}(x)|$, for every set $x$, and (as a consequence (3)), (4) the least fixed point of $\mathcal{P}$ is a proper class. Let us call an operator $\Gamma$, having the above properties (1)-(3) $\mathcal{P}$-like.

In [3] we addressed the questions: (a) How does $\mathcal{P}$ contribute to the generation of the class of all positive operators? (b) Are there any positive $\mathcal{P}$-like operators "independent" of $\mathcal{P}$ ?

We showed the following: (a) The class of all positive operators is generated from $\mathcal{P}$, the identity $I d$ and almost constant operators by composition, finitary $\cup, \cap$ and uniform and infinitary $U$ and $\cap$. This enables one to define strictly what a $\mathcal{P}$-independent operator is. (b) If $\Gamma$ is positive, $\mathcal{P}$-independent and bounded (i.e., defined by a bounded formula), then $\Gamma$ is not $\mathcal{P}$-like.

So the question whether there are positive, $\mathcal{P}$-independent, unbounded $\mathcal{P}$-like operators remains open. Obviously the simplest cases of unbounded operators are those with quantifier prefix $\bar{\forall} \bar{w}$ or $\bar{\exists} \bar{w}$ in their defining formula (the latter being in its canonical form). However even for these operators with such a low logical complexity - let alone those containing alternations of quantifiers - it is quite hard to check whether they are all $\mathcal{P}$-independent or not. The reason is that each proof is ad hoc, by cases, and no general method seems to be available. In this paper we examine positive unbounded operators whose defining formula has a quantifier prefix $\bar{Q} \bar{w}$ consisted (at most) only of universal quantifiers, i.e., $\bar{Q}=\bar{\forall}$ or $\bar{Q}=\emptyset$ (see lemma 1.1 below). We couldn't establish the analogous result for prefix $\bar{\exists}$. (Note that in the defining formula of $\mathcal{P}, \bar{Q}=\emptyset$.) Specifically, it is shown that every operator $\Gamma$ defined by a positive formula with prefix $\bar{\forall}$, for 
which in addition $\Gamma(\emptyset) \neq \emptyset$, is (finitely) generated from $\mathcal{P}, I d$, constant operators and certain trivial ones, extending thus the result (a) of [3]. (Obviously the operators $\Gamma$ for which $\Gamma(\emptyset)=\emptyset$ are not $\mathcal{P}$-like in a striking way, so leaving them out of consideration is no restriction at all.)

Throughout our metatheory will be GBC (Gödel-Bernays set theory). $L$ will be the language of GBC. As usual upper case letters $X, Y, S, \ldots$ denote class variables or constants, while lower case letters $x, y, a, b, u, w, \ldots$ denote set variables or constants. We recall the following definitions.

A (unary) operator (without parameters) is produced by a secondorder formula $\phi(v, S)$ of the language of set theory, where $v$ is a set variable and $S$ is a class variable. $\phi(v, S)$ gives rise to the operator $\Gamma_{\phi}$ defined by

$$
\Gamma_{\phi}(X)=\{x: \phi(x, X)\} .
$$

In general $\Gamma_{\phi}$ sends classes to classes but, mainly, we shall be interested in those $\phi$ such that for every set $a, \Gamma_{\phi}(a)$ is a set. Such an operator will be called set-theoretic, or a set-operator.

An operator $\Gamma_{\phi}$ is said to be monotone if $X \subseteq Y \Rightarrow \Gamma_{\phi}(X) \subseteq$ $\Gamma_{\phi}(Y)$. (In the preceding notation, lower case letters $x, y$ denote sets, while upper case $X, Y$ denote classes.) In order for $\Gamma_{\phi}$ to be monotone it suffices for $\phi$ to be positive in $S$. $\phi$ is positive in $S$ if it is constructed by formulas not containing $S$ and atomic formulas $u \in S$ using only the logical operations $\wedge, \vee, \exists$ and $\forall$. (See e.g. [2].)

$X$ is a fixed point of $\Gamma_{\phi}$ if $\Gamma_{\phi}(X)=X$. Every monotone operator $\Gamma_{\phi}$ has a least fixed point denoted $I_{\phi}$. Moreover it is well-known that $I_{\phi}=\bigcup_{\alpha \in O n} I_{\phi}^{\alpha}$, where

$$
I_{\phi}^{0}=\emptyset, \quad I_{\phi}^{\alpha+1}=\Gamma_{\phi}\left(I_{\phi}^{\alpha}\right), \quad I_{\phi}^{\alpha}=\bigcup_{\beta<\alpha} I_{\phi}^{\beta}, \quad \text { for limit } \alpha .
$$

Moschovakis [2] has discovered a canonical form for positive formulas.

Lemma 1.1 (Moschovakis) Let $\phi(v, S)$ be a positive formula of $L$. Then there is a quantifier-free and $S$-free formula $\theta(v, \bar{w}, u)$, where $\bar{w}=\left(w_{1}, \ldots, w_{m}\right)$, and a string of quantifiers $\bar{Q}=\left(Q_{1}, \ldots, Q_{m}\right)$ such that, for every $x$ and every class $X \neq V$,

$$
\phi(x, X) \Longleftrightarrow(\bar{Q} \bar{w})(\forall u)(\theta(x, \bar{w}, u) \vee u \in X) .
$$


Proof. See [2], pp. 57-58.

By 1.1 we may assume that every positive formula has the form

$$
\phi(v, S):=(\bar{Q} \bar{w})(\forall u)(\theta(v, \bar{w}, u) \vee u \in S) .
$$

We shall refer to (2) as the canonical form of $\phi$. The string of quantifiers $\bar{Q}$ in the above form measures the complexity of $\phi$.

Let

$\mathcal{O}=\{\Gamma: \Gamma$ is positive operator of the language of set theory $\}$.

The main operation in $\mathcal{O}$ is composition, but also finite meets and unions are natural natural operations under which $\mathcal{O}$ is closed. Given $\Gamma_{1}, \ldots, \Gamma_{n}$, let $\Gamma_{1} \cup \cdots \cup \Gamma_{n}, \Gamma_{1} \cap \cdots \cap \Gamma_{n}$, be the operators defined by

$$
\begin{aligned}
& \left(\Gamma_{1} \cup \cdots \cup \Gamma_{n}\right)(X)=\Gamma_{1}(X) \cup \cdots \cup \Gamma_{n}(X), \\
& \left(\Gamma_{1} \cap \cdots \cap \Gamma_{n}\right)(X)=\Gamma_{1}(X) \cap \cdots \cap \Gamma_{n}(X) .
\end{aligned}
$$

Clearly if $\Gamma_{1}, \ldots, \Gamma_{n}$ are positive then so are $\Gamma_{1} \cup \cdots \cup \Gamma_{n}$ and $\Gamma_{1} \cap$ $\cdots \cap \Gamma_{n}$.

Definition 1.2 A positive $\Gamma$ is said to be a $\forall$-operator if it is defined by a formula $\phi(v, S)$ with canonical form $(\bar{Q} \bar{w})(\forall u)(\theta(v, \bar{w}, u) \vee u \in S)$, where $\bar{Q}=\bar{\forall}$, or $\bar{Q}=\emptyset$.

In the class of all operators the constant ones is natural to play a significant role.

Definition 1.3 $\Gamma$ is said to be constant if there is a class $A$ such that $\Gamma(x)=A$ for every set $x$. We denote this operator by $C_{A}$.

Apart from constant another kind of trivial operators are those $\Gamma$ for which $\Gamma(\emptyset)$ is a proper class.

Definition 1.4 $\Gamma$ is said to be big if $\Gamma(\emptyset)$ is a proper class. 


\section{$2 \quad \forall$-operators}

In this section we shall prove the following

Theorem 2.1 Let $\Gamma_{\phi}$ be a $\forall$-operator. Then either $I_{\phi}=\emptyset$, or $\Gamma_{\phi}=$ $\Gamma_{1} \cap \cdots \cap \Gamma_{m}$, where at most two of the $\Gamma_{i}$ 's are big, while the rest $\Gamma_{i}$ are of the form $\Gamma_{i}=\mathcal{P}^{k_{i}}\left(I d \cup C_{V_{r_{i}}}\right) \cup I d$ for some $k_{i}, r_{i} \geq 0$.

We consider first the case where $\bar{\forall}=\emptyset$, i.e., when $\phi(v, S) \Longleftrightarrow$ $(\forall u)(\theta(v, u) \vee u \in S)$..

Proposition 2.2 Let $\phi(v, S) \Longleftrightarrow(\forall u)(\theta(v, u) \vee u \in S)$.

(i) If $u$ does not occur in $\theta$, then $\Gamma_{\phi}=C_{A}$ for some $A$.

(ii) If $u$ occurs in $\theta$, then $\Gamma_{\phi}=C_{\emptyset}$, or $\Gamma_{\phi}=C_{V}$, or $\Gamma_{\phi}=\mathcal{P}$ or $\Gamma_{\phi}=I d$, or $\Gamma_{\phi}=\mathcal{P} \cup I d$.

Proof. (i) If $u$ does not occur in $\theta$, then for every $X \neq V, \phi(x, X)=$ $\theta(x) \vee(\forall u)(u \in X) \Leftrightarrow \theta(x)$. So for every $X, \Gamma_{\phi}(X)=\{x: \theta(x)\}=A$. Thus $\Gamma_{\phi}=C_{A}$ is constant.

(ii) Let $u$ occur in $\theta$. If $\theta \Leftrightarrow \top$, then $\Gamma_{\phi}=C_{V}$ and if $\theta \Leftrightarrow \perp$, then $\Gamma_{\phi}=C_{\emptyset}$

Suppose $\theta$ contains both variables $u, v$ and $\theta \Leftrightarrow \top, \perp$. Then $\theta$ is a $\{\vee, \wedge\}$-combination of the formulas $u \in v, v \in u, u=v, u \notin v, v \notin u$, $u \neq v$ (as well as of the formulas $u=u, u \in u, v=v, v \in v$ and their negations; but in view of the fact that each one of them is equivalent to either $T$ or $\perp$, we can ignore them). For every set $a$ let

$$
-a=\{x: x \notin a\}, \hat{a}=\{x: a \in x\},-\hat{a}=\{x: a \notin x\} .
$$

Clearly $-a, \hat{a},-\hat{a}$ are proper classes for all sets $a$. We have the following cases for atomic $\theta$ :

Case 1. $\theta=(u \in v)$. Then for any set $a, \Gamma_{\phi}(a)=\{x:(\forall u)(u \notin$ $x \Rightarrow u \in a)\}=\{x:-x \subseteq a\}=\emptyset$. Thus $\Gamma_{\phi}=C_{\emptyset}$.

Case 2. $\theta=(v \in u)$. Then for any set $a, \Gamma_{\phi}(a)=\{x:(\forall u)(x \notin$ $u \Rightarrow u \in a)\}=\{x:-\hat{x} \subseteq a\}=\emptyset$. Thus $\Gamma_{\phi}=C_{\emptyset}$.

Case 3. $\theta=(u=v)$. Then $\Gamma_{\phi}(a)=\{x:(\forall u)(u \neq x \Rightarrow u \in a)\}=$ $\{x:-\{x\} \subseteq a\}=\emptyset$. Thus $\Gamma_{\phi}=C_{\emptyset}$.

Case 4. $\theta=(u \notin v)$. Then for all $X, \Gamma_{\phi}(X)=\{x:(\forall u)(u \in x \Rightarrow$ $u \in X)\}=\{x: x \subseteq X\}=\mathcal{P}(X)$. Hence $\Gamma_{\phi}=\mathcal{P}$.

Case 5. $\theta=(v \notin u)$. Then for all $a, \Gamma_{\phi}(a)=\{x:(\forall u)(x \in u \Rightarrow$ $u \in a)\}=\{x: \hat{x} \subseteq a\}=\emptyset$. Thus $\Gamma_{\phi}=C_{\emptyset}$. 
Case 6. $\theta=(u \neq v)$. Then for all $X, \Gamma_{\phi}(X)=\{x:(\forall u)(u=x \Rightarrow$ $u \in X)\}=X$. Hence $\Gamma_{\phi}=I d$.

We come now to the case when $\theta$ is not atomic. Let $\Sigma=\{u \in$ $v, v \in u, u=v, u \notin v, v \notin u, u \neq v\}$. Let $\eta_{0}:=(u \in v \vee v \in u \vee u=v)$, $\eta_{1}:=(u \in v \vee v \in u), \eta_{2}=(u \in v \vee u=v)$ and $\eta_{3}=(v \in u \vee u=v)$. Let $\Sigma^{*}=\Sigma \cup\left\{\eta_{i}, \neg \eta_{i}: i \leq 3\right\} \cup\{\top, \perp\}$.

Claim. If $\theta$ is a $\{\vee, \wedge\}$-combination of formulas of $\Sigma$, then there is $\sigma \in \Sigma^{*}$ such that $\theta \Leftrightarrow \sigma$.

Proof. By induction on the length of $\theta$. Let $\theta$ be a $\{\vee, \wedge\}-$ combination of formulas $\Sigma$ for which the claim holds. It suffices to prove the claim for $\theta \vee \sigma$ and $\theta \wedge \sigma$ for every $\sigma \in \Sigma$. By the induction hypothesis, $\theta \Leftrightarrow \tau$ for some $\tau \in \Sigma^{*}$, so we have to examine all the combinations $\tau \vee \sigma$ and $\tau \wedge \sigma$, with $\tau \in \Sigma^{*}$ and $\sigma \in \Sigma$, taking into account certain facts imposed by the foundation axiom of ZF. For instance $(v \in u \wedge u=v) \Leftrightarrow \perp,(u \in v \vee v \notin u) \Leftrightarrow v \notin u$, etc. The complete checking of all cases $\tau \vee \sigma$ and $\tau \wedge \sigma$, for $\tau \in \Sigma^{*}$ and $\sigma \in \Sigma$ is left to the patient reader. This completes the proof of the Claim.

In view of the Claim it remains to examine the operators $\Gamma_{\phi}$ for formulas $\phi=(\forall u)(\theta(v, u) \vee u \in S)$, where $\theta \in\left\{\eta_{i}, \neg \eta_{i}: i \leq 3\right\} \cup\{\top, \perp\}$. $\theta=\top, \perp$ have already been considered above. So we consider the remaining formulas:

Case 7. $\theta=\eta_{0}$. Then $\Gamma_{\phi}(a)=\{x:(\forall u)(u \notin x \wedge x \notin u \wedge x \neq$ $u \Rightarrow u \in a)\}=\{x:-x \cap-\hat{x} \cap-\{x\} \subseteq a\}$. But clearly for every $x$, $-x \cap-\hat{x} \cap-\{x\}$ is a proper class. Hence $\Gamma_{\phi}(a)=\emptyset$, i.e., $\Gamma_{\phi}=C_{\emptyset}$.

Case 8. $\theta=\neg \eta_{0}$. Then $\Gamma_{\phi}(\emptyset)=\{x:(\forall u)(u \in x \vee x \in u \vee x=$ $u \Rightarrow u \in a)\}=\{x: x \cup \hat{x} \cup\{x\} \subseteq a\}=\emptyset$. Thus $\Gamma_{\phi}=C_{\emptyset}$.

Case 9. $\theta=\eta_{1}$. Then $\Gamma_{\phi}(a)=\{x:-x \cap-\hat{x} \subseteq a\}=\emptyset$. So $\Gamma_{\phi}=C_{\emptyset}$.

Case 10. $\theta=\neg \eta_{1}$. Then $\Gamma_{\phi}(a)=\{x: x \cup \hat{x} \subseteq a\}=\emptyset$. Thus $\Gamma_{\phi}=C_{\emptyset}$.

Case 11. $\theta=\eta_{2}$. Then $\Gamma_{\phi}(a)=\{x:-x \cap-\{x\} \subseteq a\}=\emptyset$. So $\Gamma_{\phi}=C_{\emptyset}$.

Case 12. $\theta=\neg \eta_{2}$. Then for every $X, \Gamma_{\phi}(X)=\{x: x \cup\{x\} \subseteq$ $X\}=\mathcal{P}(X) \cup X$. Thus $\Gamma_{\phi}=\mathcal{P} \cup I d$.

Case 13. $\theta=\eta_{3}$. Then $\Gamma_{\phi}(a)=\{x:-\hat{x} \cap-\{x\} \subseteq a\}=\emptyset$. So $\Gamma_{\phi}=C_{\emptyset}$.

Case 14. $\theta=\neg \eta_{3}$. Then for every $a, \Gamma_{\phi}(a)=\{x: \hat{x} \cup\{x\} \subseteq a\}=\emptyset$. Thus $\Gamma_{\phi}=\emptyset$. 
Inspecting all case (1)-(14) we see that $\Gamma_{\phi}$ is either $C_{\emptyset}$, or $\mathcal{P}$ or $I d$ or $\mathcal{P} \cup I d$. It follows that the claim holds.

We come now to $\phi(v, S)=(\bar{Q} \bar{w})(\forall u)(\theta(v, \bar{w}, u) \vee u \in S)$ with $\bar{Q}=\bar{\forall}$. For simplicity we shall write $\forall \bar{w}$ instead of $\bar{\forall} \bar{w}$ and similarly for $\exists$.

Proposition 2.3 Let $\phi(v, S) \Leftrightarrow(\bar{\forall} \bar{w})(\forall u)(\theta(v, \bar{w}, u) \vee u \in S)$, where $\bar{Q}=\bar{\forall}$ and $\theta$ is a disjunction of atomic or negated atomic formula. Then either $I_{\phi}=\emptyset$, or $\Gamma_{\phi}$ is big, or $\Gamma_{\phi}=\mathcal{P}^{k}\left(I d \cup C_{V_{l}}\right)$ for some $k, l \in \mathbb{N}$, where $V_{l}=\mathcal{P}^{l}(\emptyset)$ (the l-segment of the universe).

Proof. Fix a $\phi(v, S) \Leftrightarrow(\forall \bar{w})(\forall u)(\theta(v, \bar{w}, u) \vee u \in S)$ with

$$
\theta(v, \bar{w}, u)=\sigma_{1}(v, \bar{w}, u) \vee \cdots \vee \sigma_{m}(v, \bar{w}, u),
$$

where each $\sigma_{i}$ is atomic or negated atomic. Then for every $X$,

$$
\begin{gathered}
\Gamma_{\phi}(X)=\{x:(\forall \bar{w})(\forall u)[\neg \theta(x, \bar{w}, u) \Rightarrow u \in X]\}= \\
\{x:(\forall u)[(\exists \bar{w}) \neg \theta(x, \bar{w}, u) \Rightarrow u \in X]\}= \\
\left\{x:(\forall u)\left[(\exists \bar{w})\left(\neg \sigma_{1} \wedge \cdots \wedge \neg \sigma_{m}\right) \Rightarrow u \in X\right]\right\} .
\end{gathered}
$$

In particular

$$
\Gamma_{\phi}(\emptyset)=\left\{x:(\forall \bar{w})(\forall u)\left(\sigma_{1} \vee \cdots \vee \sigma_{m}\right)\right\}
$$

We examine several cases, subcases, subsubcases etc. We call them all "cases" and enumerate them by sequences of numbers. To facilitate the reader we indicate each case by $\rightarrow$ if it is a subcase of the previous one, and $\downarrow$ if it is of equal depth as the previous one. The chart of cases and subcases is as follows:

Case 1.

$\downarrow$ Case 2 .

$\rightarrow$ Case 2.1 .

$\downarrow$ Case 2.2.

$\downarrow$ Case 2.3.

$\rightarrow$ Case 2.3.1.

$\rightarrow$ Case 2.3.1.1.

$\downarrow$ Case 2.3.1.2.

$\rightarrow$ Case 2.3.1.2.1. 
$\downarrow$ Case 2.3.1.2.2.

$\leftarrow$ Case 2.3.2.

Case 1: $\theta(v, \bar{w}, u) \Leftrightarrow \top$.

Then $(\forall v)(\forall \bar{w})(\forall u)(\theta(v, \bar{w}, u)$ is true, therefore

$$
\Gamma_{\phi}(\emptyset)=\{x:(\forall \bar{w})(\forall u)(\theta(x, \bar{w}, u)\}=V .
$$

So $\Gamma_{\phi}$ is big.

$\downarrow$ Case 2: $\theta(v, \bar{w}, u) \Leftrightarrow \top$.

Then $\neg \theta(v, \bar{w}, u) \Leftrightarrow \neg \sigma_{1}(v, \bar{w}, u) \wedge \cdots \wedge \neg \sigma_{m}(v, \bar{w}, u)$ is satisfiable. Let $\Sigma=\left\{\neg \sigma_{1}, \ldots, \neg \sigma_{m}\right\}$. Each element of $\Sigma$ is again an atomic or negated atomic formula. Call graph of $\Sigma$ the directed graph $G(\Sigma)$ defined as follows: The set of nodes of $G(\Sigma)$ is the set $W=\left\{v, w_{1}, \ldots, w_{n}, u\right\}$ of variables occurring in the formulas of $\Sigma$ with the following provision: Let $\alpha, \beta \in W$. If $\alpha=\beta$ is a formula of $\Sigma$, then the nodes of $G(\Sigma)$ corresponding to these variables coincide. $\alpha \rightarrow \beta$ is an edge of $G(\Sigma)$ if there are $\alpha_{1}, \ldots, \alpha_{k}, \beta_{1}, \ldots, \beta_{l}$ such that

$$
\begin{gathered}
\left\{\alpha=\alpha_{1}, \alpha_{1}=\alpha_{2}, \ldots, \alpha_{k-1}=\alpha_{k}\right\} \cup \\
\left\{\beta=\beta_{1}, \beta_{1}=\beta_{2}, \ldots, \beta_{l-1}=\beta_{l}\right\} \cup\left\{\beta_{l} \in \alpha_{k}\right\} \subseteq \Sigma .
\end{gathered}
$$

In view of this and the fact that the cases considered below concern the various forms of the graph $G(\Sigma)$, for the rest of the proof we may ignore equalities and their negations, i.e., we may assume that $\sigma_{i}$ are only formulas of the form $\alpha_{i} \in \alpha_{j}$ and $\alpha_{i} \notin \alpha_{j}$. Further, for every node $\alpha$, let the restrictions of $\alpha$ be the set

$$
r(\alpha)=\{\beta:(\alpha \notin \beta) \in \Sigma \vee(\beta \notin \alpha) \in \Sigma \vee(\alpha \neq \beta) \in \Sigma\} .
$$

Observe that, since $\neg \sigma_{1} \wedge \cdots \wedge \neg \sigma_{m}$ is satisfiable, so is $\Sigma$. Consequently $G(\Sigma)$ contains no cycles, so each path in $G(\Sigma)$ has a terminal node. A decoration of $G(\Sigma)$ is a mapping $d: W \rightarrow V$ such that (a) if $\alpha \rightarrow \beta$ is in $G(\Sigma)$, then $d(\beta) \in d(\alpha)$, and (b) if $\alpha \notin \beta$, $\beta \notin \alpha, \alpha \neq \beta$ are in $r(\alpha)$, then $d(\alpha) \notin d(\beta), d(\beta) \notin d(\alpha)$ and $d(\alpha) \neq d(\beta)$, respectively. It follows immediately that if $d$ is a decoration of $G(\Sigma)$, then $d(v), d\left(w_{1}\right), \ldots, d\left(w_{n}\right), d(u)$ make true all $\neg \sigma_{i}$, hence $\neg \theta\left(d(v), d\left(w_{1}\right), \ldots, d\left(w_{n}\right), d(u)\right)$ is true. Also since $\neg \theta$ is satisfiable, there is already a decoration for $G(\Sigma)$.

$\rightarrow$ Case 2.1: $v$ is a terminal node of $G(\Sigma)$. 
(Equivalently, there are $\alpha_{1}, \ldots, \alpha_{l} \in W$ such that $\left\{v \in \alpha_{1}, \alpha_{1} \in\right.$ $\left.\alpha_{2}, \ldots, \alpha_{l-1} \in \alpha_{l}\right\} \subseteq \Sigma$. Or, equivalently, $\theta$ contains a subformula of the form $v \notin \alpha_{1} \vee \alpha_{1} \notin \alpha_{2} \vee \cdots \vee \alpha_{l-1} \notin \alpha_{l}$.)

Claim 1. For every $x$ there is a decoration $d$ of $G(\Sigma)$ such that $d(v)=x$. Consequently, $(\forall v)(\exists \bar{w})(\exists u) \neg \theta(v, \bar{w}, u)$ is true. Hence, by (3),

$$
\Gamma_{\phi}(\emptyset)=\{x:(\forall \bar{w})(\forall u) \theta(x, \bar{w}, u)\}=\emptyset .
$$

So $I_{\phi}=\emptyset$.

Proof. Define a rank for the elements of $W$ as follows: First set $\operatorname{rank}(\alpha)=\infty$, if $\alpha$ is an isolated node of $G(\Sigma)$. Every non-isolated node belongs to some path. So let $\operatorname{rank}(\alpha)=0$ if $\alpha$ is a terminal node, and $\operatorname{rank}(\alpha)=n+1$ if there is $\beta$ such that $\alpha \rightarrow \beta$ belongs to $G(\Sigma)$ and $\operatorname{rank}(\beta)=n$. Let $W_{i}=\{\alpha: \operatorname{rank}(\alpha)=i\}$, for $i=1, \ldots, t, \infty$, be the levels of $W$.

We define $d$ on $W_{i}$ by induction on $i$. By assumption $v \in W_{0}$. Let $\alpha_{j}, j \leq p$, be an enumeration of $W_{0}$ with $\alpha_{0}=v$. Set $d(v)=x$ and suppose $d\left(\alpha_{k}\right)$ are defined for $k<j$. Then set $d\left(\alpha_{j}\right)$ to be any set $y$ which respects the restrictions of $\alpha_{j}$ with respect to the so far defined sets $d\left(\alpha_{k}\right), k<j$. That is, if $\alpha_{k} \in d\left(\alpha_{j}\right)$ and $\alpha_{j} \notin \alpha_{k}, \alpha_{k} \notin \alpha_{j}$ or $\alpha_{j} \neq \alpha_{k}$, then we choose $y$ so that $y \notin d\left(\alpha_{k}\right), d\left(\alpha_{k}\right) \notin y$ or $y \neq d\left(\alpha_{k}\right)$. Obviously this choice is always possible.

Suppose we have defined $d$ for the elements of $W_{i}$, let $\alpha_{j}, j \leq p$, be an enumeration of $W_{i+1}$ and suppose $d\left(\alpha_{k}\right)$ are defined for $k<j$. Let $\beta_{1}, \ldots, \beta_{s}$ be the children nodes of $\alpha_{j}$. Clearly $d\left(\beta_{1}\right), \ldots, d\left(\beta_{s}\right)$ are defined. So it suffices to set $d\left(a_{j}\right)=y$ such that $\left\{d\left(\beta_{1}\right), \ldots, d\left(\beta_{s}\right)\right\} \subseteq y$ and $y$ respect also its restrictions with respect to the so far defined sets. Such a choice of $y$ is again always possible.

Finally, for any two $\gamma_{1}, \gamma_{2} \in W_{\infty}$, some of the following are in $\Sigma$ : $\gamma_{1} \notin \gamma_{2}, \gamma_{2} \notin \gamma_{1}, \gamma_{1} \neq \gamma_{2}, \gamma_{i} \notin \alpha, \alpha \notin \gamma_{i}, \gamma_{i} \neq \alpha$, where $\alpha \in W_{i}, i \leq t$. Since $d(\alpha)$ are already been defined, it suffices to check that any finite number of formulas of the form $\gamma_{1} \notin \gamma_{2}, \gamma_{2} \notin \gamma_{1}, \gamma_{1} \neq \gamma_{2}, \gamma_{i} \notin d(\alpha)$, $d(\alpha) \notin \gamma_{i}, \gamma_{i} \neq d(\alpha)$ is satisfiable. But this is obvious due to the size of co-sets. This completes the proof of the Claim 1.

$\downarrow$ Case 2.2: $v$ is an isolated node of $G(\Sigma)$.

(Equivalently, for every $\alpha \in W, \theta$ does not contain as a subformula neither $v \notin \alpha$, nor $\alpha \notin v$.)

Inspecting the proof of case 2.1 we easily deduce that if $v$ is isolated and we set $d(v)=x$, then we can extend $d$ on the whole $W$. Thus 
again $(\forall v)(\exists \bar{w})(\exists u) \neg \theta(v, \bar{w}, u)$ is true, and therefore $\Gamma_{\phi}(\emptyset)=\emptyset=I_{\phi}$, i.e., the least fixed point is a set.

$\downarrow$ Case 2.3: $v$ is neither a terminal nor an isolated node of $G(\Sigma)$.

In this case $G(\Sigma)$ contains paths of the form $v \rightarrow \alpha_{1} \rightarrow \alpha_{2} \rightarrow$ $\cdots \rightarrow \alpha_{r}$ with $r \geq 1$. Let $G(v)$ be the subgraph of $G(\Sigma)$ consisting of the paths starting at the node $v$. This is a rooted graph with root $v$. For every set $x$, let $E(x)$ be the $\in$-graph of $x$, i.e., the directed graph whose nodes are the elements of $T C(x) \cup\{x\}$ and edges $y \rightarrow z$ whenever $z \in y . E(x)$ is also rooted. Write $G(v) \preceq E(x)$ if $E(x)$ contains a subgraph with the same root $x$, isomorphic to $G(v)$.

Claim 2. If for a set $x, G(v) \npreceq E(x)$, then $(\forall \bar{w})(\forall u) \theta(x, \bar{w}, u)$. Therefore $x \in \Gamma_{\phi}(\emptyset)$.

Proof. Clearly, if there is a decoration $d$ of $G(\Sigma)$ with $d(v)=x$, then necessarily $E(x)$ must contain a subgraph isomorphic to $G(v)$ with the same root. So if $G(v) \npreceq E(x)$, then there is no decoration $d$ of $G(\Sigma)$ such that $d(v)=x$. Therefore $\neg(\exists \bar{w})(\exists u) \neg \theta(x, \bar{w}, u)$. Hence $(\forall \bar{w})(\forall u) \theta(x, \bar{w}, u)$. This completes the proof of Claim 2 .

$\rightarrow$ Case 2.3.1: $G(v)$ is non-branching, i.e., it consists of a single path $v \rightarrow \alpha_{1} \rightarrow \alpha_{2} \rightarrow \cdots \rightarrow \alpha_{r}$ with $r \geq 1$.

$\rightarrow$ Case 2.3.1.1: $u \notin\left\{\alpha_{1}, \ldots, \alpha_{r}\right\}$.

Then we may assume that $\alpha_{i}=w_{i}$ and

$$
\neg \theta \Leftrightarrow\left(w_{r} \in w_{r-1}\right) \wedge \cdots \wedge\left(w_{2} \in w_{1}\right) \wedge\left(w_{1} \in v\right) \wedge \delta(u, v, \bar{w}),
$$

where $\delta(u, v, \bar{w})$ is a conjunction of negated atoms. If some conjunct of $\delta$ is implied by $\left(w_{r} \in w_{r-1}\right) \wedge \cdots \wedge\left(w_{2} \in w_{1}\right) \wedge\left(w_{1} \in v\right)$, we can ignore it. So we may assume that every conjunct of $\delta$ is not implied by the last formula. Therefore they will be of the form:

$w_{i} \notin w_{j}$, for $i>j+1$,

$u \notin w_{i}$, for $i<r-1$,

$u \notin x$,

$w_{i} \notin v$, for $i>1$.

Thus in general

$$
\delta=\left(u \notin w_{i}\right) \wedge(u \notin x) \wedge\left(w_{j} \notin w_{k}\right) \wedge\left(w_{l} \notin v\right) .
$$

Then

$$
\Gamma_{\phi}(\emptyset)=\left\{x:(\forall \bar{w})(\forall u)\left[w_{r} \notin w_{r-1} \vee \cdots \vee w_{2} \notin w_{1} \vee w_{1} \notin x \vee\right.\right.
$$


$\left.\left.\left(u \in w_{i}\right) \vee(u \in x) \vee\left(w_{j} \in w_{k}\right) \vee\left(w_{l} \in x\right)\right]\right\}$.

Let $x \in \Gamma_{\phi}(\emptyset)$. Then

$$
\begin{gathered}
(\forall \bar{w})(\forall u)\left[w_{r} \in w_{r-1} \wedge \cdots \wedge w_{2} \in w_{1} \wedge w_{1} \in x \Rightarrow\right. \\
\left.\left.\left(u \in w_{i}\right) \vee(u \in x) \vee\left(w_{j} \in w_{k}\right) \vee\left(w_{l} \in x\right)\right]\right\} .
\end{gathered}
$$

Let $\bar{w}^{\prime}=\bar{w}-\left\{w_{j}, w_{l}\right\}$. Then the above is written equivalently,

$\left(\forall \bar{w}^{\prime}\right)\left(\forall w_{j} \notin w_{k}\right)\left(\forall w_{l} \notin x\right)(\forall u)\left[w_{r} \in w_{r-1} \wedge \cdots \wedge w_{2} \in w_{1} \wedge w_{1} \in x \Rightarrow\right.$

$$
\left.\left.\left(u \in w_{i}\right) \vee(u \in x)\right]\right\} .
$$

A moment's inspection shows that this formula is false, because for each value $a$ of $w_{i}$, the set $a \cup x$ must contain class many elements. Therefore for every $x, x \notin \Gamma_{\phi}(\emptyset)$, i.e., $\Gamma_{\phi}(\emptyset)=\emptyset$. It follows that the least fixed point of $\Gamma_{\phi}$ is $\emptyset$.

$\downarrow$ Case 2.3.1.2: $u \in\left\{\alpha_{1}, \ldots, \alpha_{r}\right\}$.

If $r=1$, then $\alpha_{1}=u$ and $\bar{w}=\emptyset$, hence also $\bar{\forall}=\emptyset$, which contradicts our assumption that $\bar{\forall} \neq \emptyset$.

So $r>1$ and let $u=\alpha_{k}$. We may assume that $\alpha_{j}=w_{j}$ for $j<k$ and $\alpha_{j}=w_{j-1}$ for $j>k$. Thus $\neg \theta$ is written

$$
\begin{gathered}
\neg \theta \Leftrightarrow\left(w_{r} \in w_{r-1}\right) \wedge \cdots \wedge\left(w_{k+1} \in u\right) \wedge \\
\left(u \in w_{k}\right) \wedge \cdots \wedge\left(w_{2} \in w_{1}\right) \wedge\left(w_{1} \in v\right) \wedge \delta(v, \bar{w}, u),
\end{gathered}
$$

where $\delta(v, \bar{w}, u)$ is as above.

$\rightarrow$ Case 2.3.1.2.1: Suppose that the implication

$$
\begin{gathered}
\left(w_{r} \in w_{r-1}\right) \wedge \cdots \wedge\left(w_{k+1} \in u\right) \wedge \\
\left(u \in w_{k}\right) \wedge \cdots \wedge\left(w_{2} \in w_{1}\right) \wedge\left(w_{1} \in v\right) \Rightarrow \delta(v, \bar{w}, u)
\end{gathered}
$$

is logically valid. Then

$$
\begin{gathered}
(\exists \bar{w}) \neg \theta(x, \bar{w}, u) \Leftrightarrow(\exists \bar{w})\left[\left(w_{r} \in w_{r-1}\right) \wedge \cdots \wedge\left(w_{k+1} \in u\right) \wedge\right. \\
\left.\left(u \in w_{k}\right) \wedge \cdots \wedge\left(w_{2} \in w_{1}\right) \wedge\left(w_{1} \in v\right)\right] .
\end{gathered}
$$

Because of separation of variables the r.h.s. of the above is written:

$$
(\exists \bar{w})\left[\left(w_{r} \in w_{r-1}\right) \wedge \cdots \wedge\left(w_{k+1} \in u\right)\right] \wedge
$$




$$
(\exists \bar{w})\left[\left(u \in w_{k}\right) \wedge \cdots \wedge\left(w_{2} \in w_{1}\right) \wedge\left(w_{1} \in v\right)\right] .
$$

Obviously $(\exists \bar{w})\left[\left(u \in w_{k}\right) \wedge \cdots \wedge\left(w_{2} \in w_{1}\right) \wedge\left(w_{1} \in x\right)\right]$ is the analytic form of the formula $u \in \cup^{k} x$ where $\cup^{k} x$ is the $k$-th iterate of the union operator $\cup$. On the other hand $(\exists \bar{w})\left[\left(w_{r} \in w_{r-1}\right) \wedge \cdots \wedge\right.$ $\left.\left(w_{k+1} \in u\right)\right]$ means $\operatorname{rank}(u) \geq r-k-1$, where $\operatorname{rank}(u)$ is the ordinary rank of the cumulative hierarchy, or equivalently, $u \notin V_{r-k-1}$. So

$(\exists \bar{w}) \neg \theta(x, \bar{w}, u) \Longleftrightarrow u \in \cup^{k} x \wedge u \notin V_{r-k-1} \Longleftrightarrow u \in\left(\cup^{k} x-V_{r-k-1}\right)$.

Observe that for any $X, Y, \cup X \subseteq Y \Leftrightarrow X \subseteq \mathcal{P}(Y)$. And, inductively, for every $k, \cup^{k} X \subseteq Y \Leftrightarrow X \subseteq \mathcal{P}^{k}(Y)$. Also $X-Y \subseteq Z \Leftrightarrow X \subseteq Y \cup Z$. So

$$
\begin{gathered}
\Gamma_{\phi}(X)=\left\{x:\left(\cup^{k} x-V_{r-k-1}\right) \subseteq X\right\}= \\
\left\{x: \cup^{k} x \subseteq\left(X \cup V_{r-k-1}\right)\right\}=\left\{x: x \subseteq \mathcal{P}^{k}\left(X \cup V_{r-k-1}\right)\right\} .
\end{gathered}
$$

Therefore $\Gamma_{\phi}(X)=\mathcal{P}^{k+1}\left(X \cup V_{r-k-1}\right)$, i.e., $\Gamma_{\phi}=\mathcal{P}^{k+1}\left(I \cup C_{V_{r-k-1}}\right)$.

$\downarrow$ Case 2.3.1.2.2: Suppose that the implication

$$
\begin{gathered}
\left(w_{r} \in w_{r-1}\right) \wedge \cdots \wedge\left(w_{k+1} \in u\right) \wedge \\
\left(u \in w_{k}\right) \wedge \cdots \wedge\left(w_{2} \in w_{1}\right) \wedge\left(w_{1} \in v\right) \Rightarrow \delta(v, \bar{w}, u)
\end{gathered}
$$

is not logically valid. We shall prove the following:

Claim 3. In this case $O n \subseteq \Gamma_{\phi}(\emptyset)$, hence $\Gamma_{\phi}(\emptyset)$ is a proper class. So $\Gamma_{\phi}$ is big.

Proof. Recall that in general $u=\alpha_{k}$. For simplicity we shall assume that $u=\alpha_{r}$. The adaptation of the proof for $u=\alpha_{k}$ is easy. Inspecting the proof of the previous case, just observe that the difference consists in having $x \subseteq \mathcal{P}(X)$ instead of $x \subseteq \mathcal{P}\left(X \cup V_{r-k-1}\right)$. The difference does not affect the truth of our claim. So let

$$
\neg \theta=\left(u \in w_{r-1}\right) \wedge \cdots \wedge\left(w_{2} \in w_{1}\right) \wedge\left(w_{1} \in v\right) \wedge \delta,
$$

and

$$
\left(u \in w_{r-1}\right) \wedge \cdots \wedge\left(w_{2} \in w_{1}\right) \wedge\left(w_{1} \in v\right) \Rightarrow \delta
$$

is not logically valid. By the last assumption, at least one of the conjuncts of $\delta$ is not logically implied by $\left(u \in w_{r-1}\right) \wedge \cdots \wedge\left(w_{2} \in\right.$ $\left.w_{1}\right) \wedge\left(w_{1} \in v\right)$. As above $\delta$ will contain some conjunct of the following kinds: 
(a) $w_{i} \notin w_{j}$, for $r>i>j+1 \geq 2$,

(b) $u \notin w_{i}$, for $r-1>i$,

(c) $u \notin x$,

(d) $w_{i} \notin x$, for $r>i>1$.

We shall examine these subcases one by one.

Case (a): Let $\delta$ contain $w_{i} \notin w_{j}$, for some $r>i>j+1 \geq 2$. Let

$\theta^{\prime}(x, \bar{w}, u)=\left(u \notin w_{r-1}\right) \vee \cdots \vee\left(w_{2} \notin w_{1}\right) \vee\left(w_{1} \notin x\right) \vee\left(w_{i} \in w_{j}\right)$

and

$$
\phi^{\prime}(x, S)=(\forall \bar{w})(\forall u)\left(\theta^{\prime}(x, \bar{w}, u) \vee u \in S\right) .
$$

Since $\phi^{\prime}(x, S) \Rightarrow \phi(x, S), \Gamma_{\phi^{\prime}}(X) \subseteq \Gamma_{\phi}(X)$ for every $X$, it suffices to prove that $O n \subseteq \Gamma_{\phi^{\prime}}(\emptyset)$.

We have

$$
\Gamma_{\phi^{\prime}}(X)=\left\{x:(\forall u)\left[(\exists \bar{w}) \neg \theta^{\prime}(x, \bar{w}, u) \Rightarrow u \in X\right]\right\},
$$

and

$\neg \theta^{\prime}(v, \bar{w}, u)=\left(u \in w_{r-1}\right) \wedge \cdots \wedge\left(w_{2} \in w_{1}\right) \wedge\left(w_{1} \in v\right) \wedge\left(w_{i} \notin w_{j}\right)$.

For any $x, r$, and $r>i>j+1 \geq 2$, define the following conditional iterated union:

$\cup_{i, j}^{r-1} x=\left\{u:(\exists \bar{w})\left[\left(u \in w_{r-1}\right) \wedge \cdots \wedge\left(w_{2} \in w_{1}\right) \wedge\left(w_{1} \in x\right) \wedge\left(w_{i} \notin w_{j}\right)\right]\right\}$.

Then $\Gamma_{\phi^{\prime}}(X)$ is written

$$
\Gamma_{\phi^{\prime}}(X)=\left\{x: \cup_{i, j}^{r-1} x \subseteq X\right\}
$$

Now we have

$$
\begin{gathered}
u \in \cup_{i, j}^{r-1} x \Leftrightarrow(\exists \bar{w})\left[\left(u \in w_{r-1}\right) \wedge \cdots \wedge\left(w_{2} \in w_{1}\right) \wedge\left(w_{1} \in x\right) \wedge\left(w_{i} \notin w_{j}\right)\right] \Leftrightarrow \\
(\exists t)(\exists s)\left[u \in \cup^{r-i-1} t \wedge t \in\left(\cup^{i-j} s-s\right) \wedge s \in \cup^{j} x\right] .
\end{gathered}
$$

So for all $X$ and $x$,

$$
\left.\cup_{i, j}^{r-1} x \subseteq X \Longleftrightarrow\left(\forall s \in \cup^{j} x\right)\left(\forall t \in \cup^{i-j} s-s\right)\left[\cup^{r-i-1} t \subseteq X\right)\right] .
$$

Therefore in view of (4), 


$$
\left.x \in \Gamma_{\phi^{\prime}}(X) \Longleftrightarrow\left(\forall s \in \cup^{j} x\right)\left(\forall t \in \cup^{i-j} s-s\right)\left[\cup^{r-i-1} t \subseteq X\right)\right] .
$$

The last equivalence for $X=\emptyset$ yields

$$
\left.x \in \Gamma_{\phi^{\prime}}(\emptyset) \Longleftrightarrow\left(\forall s \in \cup^{j} x\right)\left(\forall t \in \cup^{i-j} s-s\right)\left[\cup^{r-i-1} t \subseteq \emptyset\right)\right] .
$$

For any $x \in O n$, clearly $\cup^{j} x=x$ and for every $s \in x, s$ is an ordinal again, so $\cup^{i-j} s=s$, i.e., $\cup^{i-j} s-s=\emptyset$. Therefore the right-hand side of the preceding equivalence is vacuously true for every $x \in O n$, so On $\subseteq \Gamma_{\phi^{\prime}}(\emptyset)$. This completes the proof of the case (a).

Case (b): Let $\delta$ contain $u \notin w_{i}$, for $r-1>i$. Then $\neg \theta^{\prime}(x, \bar{w}, u)=\left(u \in w_{r-1}\right) \wedge \cdots \wedge\left(w_{2} \in w_{1}\right) \wedge\left(w_{1} \in x\right) \wedge\left(u \notin w_{i}\right)$

and

$$
\Gamma_{\phi^{\prime}}(X)=\left\{x:(\forall u)\left[(\exists \bar{w}) \neg \theta^{\prime}(x, \bar{w}, u) \Rightarrow u \in X\right]\right\} .
$$

For any $x, r$, and $r-1>i$, define the following conditional iterated union:

$\cup_{i}^{r-1} x=\left\{u:(\exists \bar{w})\left[\left(u \in w_{r-1}\right) \wedge \cdots \wedge\left(w_{2} \in w_{1}\right) \wedge\left(w_{1} \in x\right) \wedge\left(u \notin w_{i}\right)\right]\right\}$.

Then $\Gamma_{\phi^{\prime}}(X)$ is written

$$
\Gamma_{\phi^{\prime}}(X)=\left\{x: \cup_{i}^{r-1} x \subseteq X\right\} .
$$

Now

$u \in \cup_{i}^{r-1} x \Leftrightarrow(\exists \bar{w})\left[\left(u \in w_{r-1}\right) \wedge \cdots \wedge\left(w_{2} \in w_{1}\right) \wedge\left(w_{1} \in x\right) \wedge\left(u \notin w_{i}\right)\right] \Leftrightarrow$ $(\exists s)\left[u \in \cup^{r-i-1} s-s \wedge s \in \cup^{i} x\right]$,

hence

$$
u \in \cup_{i}^{r-1} x \Leftrightarrow(\exists s)\left[u \in \cup^{r-i-1} s-s \wedge s \in \cup^{i} x\right],
$$

From the last equivalence and (6) we have for any $x$ and $X$,

$$
x \in \Gamma_{\phi^{\prime}}(X) \Longleftrightarrow\left(\forall s \in \cup^{i} x\right)\left(\cup^{r-i-1} s-s \subseteq X\right) .
$$

For $X=\emptyset$ the preceding relation gives

$$
x \in \Gamma_{\phi^{\prime}}(\emptyset) \Longleftrightarrow\left(\forall s \in \cup^{i} x\right)\left(\cup^{r-i-1} s-s \subseteq \emptyset\right) .
$$


As in the previous case we easily see that right-hand side of this equivalence is true for every $x \in O n$. Therefore $O n \subseteq \Gamma_{\phi^{\prime}}(\emptyset)$.

Case (c): Let $\delta$ contain $u \notin x$. Then

$\neg \theta^{\prime}(x, \bar{w}, u)=\left(u \in w_{r-1}\right) \wedge \cdots \wedge\left(w_{2} \in w_{1}\right) \wedge\left(w_{1} \in x\right) \wedge(u \notin x)$

and

$$
\Gamma_{\phi^{\prime}}(X)=\left\{x:(\forall u)\left[(\exists \bar{w}) \neg \theta^{\prime}(x, \bar{w}, u) \Rightarrow u \in X\right]\right\} .
$$

Then clearly

$(\exists \bar{w})\left[\left(u \in w_{r-1}\right) \wedge \cdots \wedge\left(w_{2} \in w_{1}\right) \wedge\left(w_{1} \in x\right) \wedge(u \notin x)\right] \Longleftrightarrow u \in \cup^{r-1} x-x$.

So for every class $X$,

$$
x \in \Gamma_{\phi^{\prime}}(X) \Longleftrightarrow \cup^{r-1} x-x \subseteq X .
$$

In particular

$$
x \in \Gamma_{\phi^{\prime}}(\emptyset) \Longleftrightarrow \cup^{r-1} x-x \subseteq \emptyset,
$$

which holds for every $x \in O n$. Therefore $O n \subseteq \Gamma_{\phi^{\prime}}(\emptyset)$.

Case (d): Let $\delta$ contain $w_{i} \notin x$, for $r>i>1$. Then

$\neg \theta^{\prime}(x, \bar{w}, u)=\left(u \in w_{r-1}\right) \wedge \cdots \wedge\left(w_{2} \in w_{1}\right) \wedge\left(w_{1} \in x\right) \wedge\left(w_{i} \notin x\right)$

and

$$
\Gamma_{\phi^{\prime}}(X)=\left\{x:(\forall u)\left[(\exists \bar{w}) \neg \theta^{\prime}(x, \bar{w}, u) \Rightarrow u \in X\right]\right\} .
$$

Let us set

$\Delta_{i}^{r-1}=\left\{u:(\exists \bar{w})\left[\left(u \in w_{r-1}\right) \wedge \cdots \wedge\left(w_{2} \in w_{1}\right) \wedge\left(w_{1} \in x\right) \wedge\left(w_{i} \notin x\right)\right.\right.$.

Then $\Gamma_{\phi^{\prime}}(X)$ is written

$$
\Gamma_{\phi^{\prime}}(X)=\left\{x: \Delta_{i}^{r-1} x \subseteq X\right\} .
$$

Now as before it is easy to see that

$$
\Delta_{i}^{r-1}=\bigcup\left\{\cup^{r-i-1} s: s \in \cup^{i} x-x\right\} .
$$

Hence for every class $X$,

$$
\Delta_{i}^{r-1} \subseteq X \Longleftrightarrow \cup^{r-i-1} s \subseteq X, \forall s \in \cup^{i} x-x .
$$


Thus

$$
x \in \Gamma_{\phi^{\prime}}(X) \Longleftrightarrow\left(\forall s \in \cup^{i} x-x\right)\left(\cup^{r-i-1} s \subseteq X\right) .
$$

For $X=\emptyset$ we get

$$
x \in \Gamma_{\phi^{\prime}}(\emptyset) \Longleftrightarrow\left(\forall s \in \cup^{i} x-x\right)\left(\cup^{r-i-1} s \subseteq \emptyset\right) .
$$

For $x \in O n, \cup^{i} x-x=\emptyset$. So the right- hand side of the last equivalence is vacuously true, hence $O n \subseteq \Gamma_{\phi^{\prime}}(\emptyset)$.

This completes the proof of case (d) and the proof of Claim 3.

$\leftarrow$ Case 2.3.2: Suppose $G(v)$ is branching.

Without loss of generality, assume that $v$ is a branching node. (The adaptation of the argument for any other branching node is easy.) Now it is easy to see that there are class many sets $x$ such that $G(v) \npreceq E(x)$. Indeed, for any $x=\{y\}, G(v) \npreceq E(x)$ since the node $x$ of $E(x)$ is non-branching. If $X=\{x: G(v) \npreceq E(x)\}$, then by Claim $2, X \subseteq \Gamma_{\phi}(\emptyset)$, hence $\Gamma_{\phi}(\emptyset)$ is a proper class. Therefore $\Gamma_{\phi}$ is big.

This completes the checking of all possible cases and the proof. $\dashv$

Proof of Theorem 2.3. Let $\theta=\bigwedge_{i \leq k} \theta_{i}$ be the conjunctive normal form of $\theta$. Then each $\theta_{i}$ is a disjunction of atomic or negated atomic formulas. Moreover we have

$$
\begin{gathered}
\phi(v, S) \Leftrightarrow(\forall \bar{w})(\forall u)(\theta \vee u \in S) \Leftrightarrow(\forall \bar{w})(\forall u)\left(\bigwedge_{i \leq k} \theta_{i} \vee u \in S\right) \Leftrightarrow \\
(\forall \bar{w})(\forall u) \bigwedge_{i \leq k}\left(\theta_{i} \vee u \in S\right) \Leftrightarrow \bigwedge_{i \leq k}\left[(\forall \bar{w})(\forall u)\left(\theta_{i} \vee u \in S\right] .\right.
\end{gathered}
$$

Therefore, if $\phi_{i}(x, S):=(\forall \bar{w})(\forall u)\left(\theta_{i}(v, \bar{w}, u) \vee u \in S\right)$, then for all $X$, $\Gamma_{\phi}(X)=\bigcap_{i \leq k} \Gamma_{\phi_{i}}(X)$. If $\bar{\forall}=\emptyset$, then, by proposition 2.2 , each $\Gamma_{\phi_{i}}$ is $C_{A}$ or $\mathcal{P}$, or $I d$, or $\mathcal{P} \cup I d$, hence it is of the form $\mathcal{P}^{k}\left(C_{r} \cup I d\right) \cup I d$.

Suppose $\bar{\forall} \neq \emptyset$. Then the distinct cases for each $\Gamma_{\phi_{i}}$ are the following: $1,2.1,2.2,2.3 .1 .1,2.3 .1 .2 .1,2.3 .1 .2 .2,2.3 .2$. If some of the $\theta_{i}$ belongs to the case 1 of proposition 2.3, i.e., $\theta_{i} \Leftrightarrow \top$, we just ignore it. If some of the $\theta_{i}$ belongs to the case 2.1 or 2.2 or 2.3.1.1, then $\Gamma_{\phi_{i}}(\emptyset)=\emptyset$, hence also $\Gamma_{\phi}(\emptyset)=\emptyset$ and so $I_{\phi}=\emptyset$.

It remains to examine the cases 2.3.1.2.1, 2.3.1.2.2, and 2.3.2. If $\Gamma_{\phi_{i}}$ is as in case 2.3.1.2.1, then $\Gamma_{\phi_{i}}(X)=\mathcal{P}^{k}\left(X \cup V_{r}\right)$ for some $k, r$, 
i.e., $\Gamma_{\phi_{i}}=\mathcal{P}^{k}\left(I \cup C_{V_{r}}\right)$, which is an instance of the general form of the theorem. If $\Gamma_{\phi_{i}}$ is as in case 2.3.1.2.2, then $O n \subseteq \Gamma_{\phi_{i}}(\emptyset)$. Finally if $\Gamma_{\phi_{i}}$ is as in case 2.3.2, then $G_{\theta_{i}}(v)$ is a branching graph. In such a case, there are $l, p, s$ (some of them may be 0 ) such that

$\Gamma_{\phi}=\mathcal{P}^{k_{1}}\left(I d \cup C_{V_{r_{1}}}\right) \cap \cdots \cap \mathcal{P}^{k_{l}}\left(I d \cup C_{V_{r_{l}}}\right) \cap\left(\Gamma_{1} \cap \cdots \cap \Gamma_{p}\right) \cap\left(\Delta_{1} \cap \cdots \cap \Delta_{s}\right)$,

where for each $i \leq p O n \subseteq \Gamma_{i}(\emptyset)$, and for each $j \leq s, \Delta_{j}$ is induced by a disjunction $\theta_{j}$, for which $G_{\theta_{j}}(v)$ is a branching graph.

Let $\Gamma=\Gamma_{1} \cap \cdots \cap \Gamma_{p}$. Since $O n \subseteq \Gamma_{i}(\emptyset)$ for every $i \leq p$, it follows that $O n \subseteq \Gamma(\emptyset)$, hence $\Gamma$ is big.

Further, since $G_{\theta_{j}}(v)$, for $j \leq s$, are branching graphs, we easily see as in case 2.3.2, that there is a proper class $X$ such that for every $x \in X, G_{\theta_{j}}(v) \npreceq E(x)$. In view of Claim 2 of proposition 2.3, $X \subseteq$ $\Delta_{j}(\emptyset)$ for all $j$. If we set $\Delta=\Delta_{1} \cap \cdots \cap \Delta_{s}$, then $X \subseteq \Delta(\emptyset)$, hence $\Delta$ is big. Therefore

$$
\Gamma_{\phi}=\mathcal{P}^{k_{1}}\left(I d \cup C_{V_{r_{1}}}\right) \cap \cdots \cap \mathcal{P}^{k_{l}}\left(I d \cup C_{V_{r_{l}}}\right) \cap \Gamma \cap \Delta,
$$

where $\Gamma, \Delta$ are big. This completes the proof of the theorem.

\section{References}

[1] S. Feferman, Why the programs for new axioms need to be questioned, Bull. Symb. Logic 6 (2000), 401-413.

[2] Y. Moschovakis, Elementary induction on abstract structures, North Holland P.C. 1974.

[3] A. Tzouvaras, What is so special with the powerset operation?, submitted. 